\title{
Correctness investigation for the suspension transport problem in coastal systems
}

\author{
Alexander I. Sukhinov ${ }^{1}$, Valentina $V$. Sidoryakina ${ }^{2, *}$ and Sofya $V$. Protsenko ${ }^{1}$ \\ ${ }^{1}$ Don State Technical University, 344000 Rostov-on-Don, Russia \\ ${ }^{2}$ Chekhov Taganrog Institute Taganrog branch of Rostov State University of Economics, Taganrog, \\ Russia
}

\begin{abstract}
This article is devoted to the confirmation the need for using a set of 3D dynamics models describing the various hydrophysical characteristics of the studied object to solve practical problems associated with the assessment of the ecological state of the water reservoirs. The present paper is devoted to the study of the three-dimensional model of transport and sedimentation of suspended matter in the coastal zone. The model takes into account such parameters as water movement, diffusionconvection, complicated bottom and shoreline geometry, lifting, transport and sedimentation of slurry. The existence and uniqueness of the solution of the corresponding indicated model of the initial-boundary value problem haas been envestigateded for two typical bottom boundary condirions. Also solution stability of the boundary-value problem in depend of functions: initial condition, boundary conditions and the righthand side in the norm $L_{2}$ for any moment of time $0<T<+\infty$, and also in the time-integral norm $L_{2}$ has been proved. The model may be basis for the construction of hydrophysics models used to describe processes in the extraction of minerals from the seabed, in the dissemination of suspensions in shelf regions.
\end{abstract}

\section{Introduction}

The great importance belongs to the protection of the aquatic environment among the factors determining the integrated development of coastal areas research. Elimination of the consequences of natural processes, such as pollution, siltation and depletion of the water areas, necessitates the study of all aspects of changes affecting the coastal waters. The maintenance of water bodies in proper condition and timely intervention in the regime of its functioning is directly connected with the increase of port capacities and the effective development of coastal infrastructure (providing access to the berths of vessels with low planting, clearing of silt and water vegetation of the coastal strip, etc.). The researches in this area require the construction of mathematical models that are quite close to real processes [1-5].

\footnotetext{
* Corresponding author: cvv9@mail.ru
} 
The paper considers a continuous mathematical model describing three-dimensional spatial processes associated with the formation, transport and gravitational sedimentation of suspensions in an aqueous medium [6-10]. The suspension transport model allows to carry out hydrophysical processes of water systems, predict the dynamics of bottom surface changes on the basis of the description of the processes of ascent, transport, deposition, and change in the concentration of suspended matter. The uniqueness of the solution of the corresponding described model of the initial-boundary value problem is proved and an a priori estimate of the solution norm is obtained depending on the integral estimates of the right-hand side, the boundary conditions and the initial condition.

\section{Continuous 3D model of diffusion-convection convection and the corresponding initial-boundary value problem}

We consider a continuous mathematical model for the propagation of suspended matter in an aqueous medium, taking into account the diffusion and convection of suspended matter, the action of gravity on the slurry, the presence of a bottom and a free surface. We will use a rectangular Cartesian coordinate system $O x y z$, where the axis $O x$ passes along the unperturbed water surface and is directed towards the sea, the axis $O z$ is directed vertically downwards. Let $h=H+\eta$ is the total depth of the water area, [m]; $H$ is the the depth at the unperturbed surface of the reservoir, $[\mathrm{m}] ; \eta$ is the elevation of the free surface relative to the geoid (sea level), [m] (Fig. 1).

Suppose that in the closure of the domain $\bar{G}=\left\{0 \leq x \leq L_{x}, 0 \leq y \leq L_{y}, 0 \leq z \leq L_{z}\right\}$ there are particles of suspended matter that are at the point $(x, y, z)$ and at the time $t$ have concentration $c=c(x, y, z, t),[\mathrm{mg} / 1] ; t$ is the time variable, [sec]. We shall also use the notation $L_{z} \equiv \max _{0 \leq x \leq L_{x}, 0 \leq y \leq L_{y}} H(x, y)$.

The equation describing the behavior of the suspended particles will look like this:

$$
\frac{\partial c}{\partial t}+\frac{\partial(u c)}{\partial x}+\frac{\partial(v c)}{\partial y}+\frac{\partial\left(\left(w+w_{g}\right) c\right)}{\partial z}=\mu_{h}\left(\frac{\partial^{2} c}{\partial x^{2}}+\frac{\partial^{2} c}{\partial y^{2}}\right)+\frac{\partial}{\partial z}\left(\mu_{v} \frac{\partial c}{\partial z}\right)+F,
$$

where $u, v, w$ are the vector components $\vec{U}$ is the velocity of the fluid, [m/s]; $w_{g}$ is the hydraulic size or particle sedimentation rate, $[\mathrm{m} / \mathrm{s}] ; \mu_{h}, \mu_{v}$ are the coefficients of horizontal and vertical turbulent diffusion of particles, respectively, $\left[\mathrm{m}^{2} / \mathrm{sec}\right] ; F$ is the power of the particle sources, $[\mathrm{mg} / 1 \mathrm{sec}]$.

The terms on the left side (except for the time derivative) of equation (1) describe the convection of particles: their transport under the action of fluid flow and gravity. The terms on the right-hand side describe the diffusion of the slurry. The vertical diffusion coefficient is chosen to be different from the horizontal diffusion coefficient due to the fact that the effect of the difference in these coefficients is often observed in different media and may be caused by various factors.

As an area $G$ consider a «sloping» to the shore «parallelepiped» $A B C D A_{1} O C_{1} D_{1}$, upper base $A_{1} O C_{1} D_{1}$ which lies on the free surface $(z=0)$, and the lower base $A B C D$ is part of the bottom surface $(z=H(x, y))$. Let $S$ is the surface $\bar{G}, \vec{n}$ is the the outer normal to the surface of the «bevelled parallelepiped». We shall assume that $\overrightarrow{U^{*}}$ is the velocity of fluid 
on the side surfaces $\bar{G}$. Together with the boundary conditions of the first kind for the particle concentration function, this makes it possible to determine the fluxes of suspended matter both towards the shore and along the coast (Fig. 1).

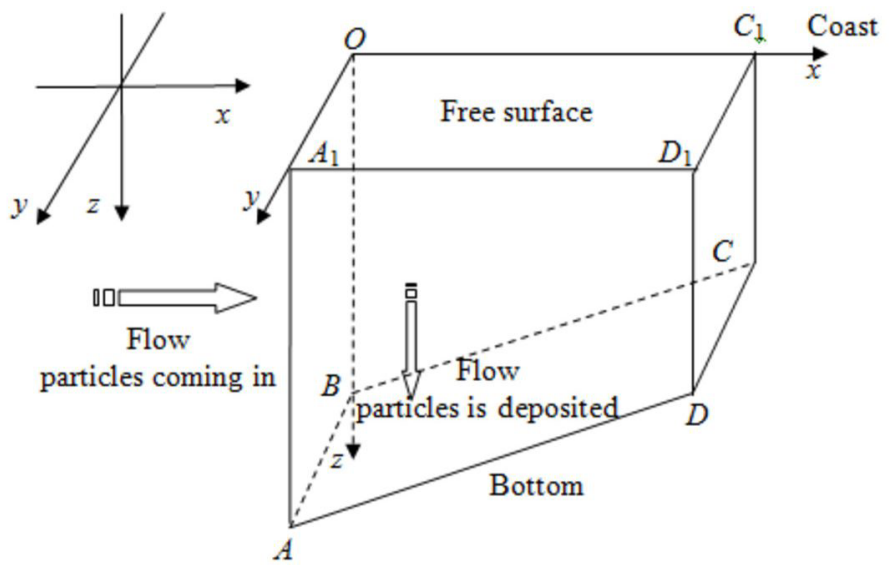

Fig. 1. The solution area for the transport of suspended matter.

Let add to the equation (1) the initial and boundary conditions.

As an initial condition with time $t=0$ accept

$$
c(x, y, z, 0) \equiv c_{0}(x, y, z) .
$$

The boundary conditions are given on the faces $A B C D A_{1} O C_{1} D_{1}$ (we set streams of suspensions both towards the shore and along the shore)

- on the faces $A A_{1} O B\left(x=0,0 \leq y \leq L_{y}, 0 \leq z \leq L_{z}\right), A A_{1} D_{1} D\left(y=L_{y}, 0 \leq x \leq L_{x}, 0 \leq z \leq L_{z}\right)$, $B O C_{1} C\left(y=0,0 \leq x \leq L_{x}, 0 \leq z \leq L_{z}\right)$

$$
c=c^{*}, \text { where } c^{*}=c^{*}(x, y, z, t), t \in[0, T] ;
$$

- on the faces $D D_{1} C_{1} C\left(x=L_{x}, 0 \leq y \leq L_{y}, 0 \leq z \leq L_{z}\right), A_{1} O C_{1} D_{1}\left(z=0,0 \leq x \leq L_{x}, 0 \leq y \leq L_{y}\right)$

$$
c=0
$$

- on the surface $A B C D\left(z=L_{z}, 0 \leq x \leq L_{x}, 0 \leq y \leq L_{y}\right)$

$$
\frac{\partial c}{\partial n}=-\frac{w_{g}}{\mu_{v}} c \text { or } \frac{\partial c}{\partial z}=-\frac{w_{g}}{\mu_{v}} c .
$$

The boundary condition (5) takes place at relatively small inclines of the bottom, that its value $\max _{A B C D} \sqrt{\left(\frac{\partial H}{\partial x}\right)^{2}+\left(\frac{\partial H}{\partial y}\right)^{2}}$ is much smaller than 1 .

In the study of combined sediment transport models, it is possible to increase the concentration of suspended particles in the bottom layer by raising the sediment particles when the shear stress is exceeded by some critical value. Then instead of the boundary condition (5) we will consider a boundary condition in the form 


$$
\frac{\partial c}{\partial z}=\alpha c, \alpha=\text { const }>0 .
$$

We will not investigate in this paper the existence of solutions of the initial-boundary value problems (1)-(5) and (1)-(4), (6). The existence of solutions of initial-boundary value problems for equations of parabolic type with lower derivatives (diffusion-convection equations) are considered, for example, in monographs [11-12].

\section{Investigation of the uniqueness of the solution of the initial-boundary problem of transport of suspended matter}

Let multiply the left and right sides of equation (1) by the function $c$ and get:

$$
c \frac{\partial c}{\partial t}+c \frac{\partial(u c)}{\partial x}+c \frac{\partial(v c)}{\partial y}+c \frac{\partial\left(\left(w+w_{g}\right) c\right)}{\partial z}=\mu_{h}\left(\frac{\partial^{2} c}{\partial x^{2}}+\frac{\partial^{2} c}{\partial y^{2}}\right)+\frac{\partial}{\partial z}\left(\mu_{v} \frac{\partial c}{\partial z}\right)+F .
$$

The left-hand side of the equality obtained may be transformed as follows:

$$
c \frac{\partial c}{\partial t}+c\left(\frac{\partial(u c)}{\partial x}+\frac{\partial(v c)}{\partial y}+\frac{\partial\left(\left(w+w_{g}\right) c\right)}{\partial z}\right)=\frac{1}{2} \frac{\partial c^{2}}{\partial t}+c \operatorname{div}(c \vec{U})=\frac{1}{2} \frac{\partial c^{2}}{\partial t}+\frac{1}{2} \operatorname{div}\left(c^{2} \vec{U}\right),
$$

where $\vec{U}=\left\|u, v, w+w_{g}\right\|^{T}$.

Taking into account (8), equation (7) can be written in the form

$$
\frac{1}{2} \frac{\partial c^{2}}{\partial t}+\frac{1}{2} \operatorname{div}\left(c^{2} \vec{U}\right)=\mu_{h} c\left(\frac{\partial^{2} c}{\partial x^{2}}+\frac{\partial^{2} c}{\partial y^{2}}\right)+c \frac{\partial}{\partial z}\left(\mu_{v} \frac{\partial c}{\partial z}\right)+c F .
$$

Then we integrate both sides of equation (9) in time on the interval $0 \leq t \leq T$ and, after that over space in the region $G$. In the first term, the order of integration is changed by Fubini's theorem. We get

$$
\begin{gathered}
\iiint_{G} \frac{1}{2}\left(\int_{0}^{T} \frac{\partial c^{2}}{\partial t} d t\right) d G+\int_{0}^{T} \frac{1}{2}\left(\iiint_{G} d i v\left(c^{2} \vec{U}\right) d G\right) d t= \\
=\int_{0}^{T}\left(\iiint_{G} c \mu_{h}\left(\frac{\partial^{2} c}{\partial x^{2}}+\frac{\partial^{2} c}{\partial y^{2}}\right) d G\right) d t+\int_{0}^{T}\left(\iiint_{G} c \frac{\partial}{\partial z}\left(\mu_{v} \frac{\partial c}{\partial z}\right) d G\right) d t+\int_{0}^{T}\left(\iiint_{G} c F d G\right) d t .
\end{gathered}
$$

The first term on the left-hand side of (10) is obviously equal to

$$
\iiint_{G} \frac{1}{2}\left(\int_{0}^{T} \frac{\partial c^{2}}{\partial t} d t\right) d G=\iiint_{G} \frac{1}{2}\left(c^{2}(x, y, z, T)-c^{2}(x, y, z, 0)\right) d G .
$$

Let us turn to the transformation of the second term on the left-hand side of (10). Taking into account the Ostrogradskii-Gauss formula and the boundary conditions (3)-(5), it may be written in the form

$$
\begin{aligned}
& \int_{0}^{T}\left(\frac{1}{2} \iiint_{G} d i v\left(c^{2} \vec{U}\right) d G\right) d t=\frac{1}{2} \int_{0}^{T}\left(\iint_{A A_{1} O B}\left(c^{*}\right)^{2}\left(\overrightarrow{U^{*}}, \vec{n}\right) d y d z\right) d t+\frac{1}{2} \iint_{0}^{T}\left(\iint_{A B C D} c^{2} w_{g} d x d y\right) d t+ \\
& +\frac{1}{2} \int_{0}^{T}\left(\int_{B O C_{1} C}\left(c^{*}\right)^{2}\left(\overrightarrow{U^{*},}, \vec{n}\right) d x d z\right) d t+\frac{1}{2} \int_{0}^{T}\left(\iint_{A A_{1} D D_{1}}\left(c^{*}\right)^{2}\left(\overrightarrow{U^{*}}, \vec{n}\right) d x d z\right) d t=-\frac{1}{2} \int_{0}^{T}\left(\iint_{A A_{1} O B}\left(c^{*}\right)^{2} u d y d z\right) d t-( \\
& -\frac{1}{2} \int_{0}^{T}\left(\int_{B O C_{1} C}\left(c^{*}\right)^{2} v d x d z\right) d t+\frac{1}{2} \int_{0}^{T}\left(\iint_{A A_{1} D D_{1}}\left(c^{*}\right)^{2} v d x d z\right) d t+\frac{1}{2} \int\left(\iint_{0}^{T} \int_{A B C D} c^{2} w_{g} d x d y\right) d t .
\end{aligned}
$$


where $\vec{U}^{*}$ is the given (known) velocity of the water medium on all faces, where boundary conditions of the Dirichlet type are given; in fact this is all the side faces, except $D D_{1} C_{1} C$ and the top cover $A_{1} O C_{1} D_{1}$, at which the concentration of suspended matter is 0 , therefore the flows through them are equal to 0 .

Let us turn to the transformation of the right-hand side of (10).

We have the equality

$$
\begin{gathered}
\iiint_{G}\left[c\left(\mu_{h} \frac{\partial}{\partial x}\left(\frac{\partial c}{\partial x}\right)+\mu_{h} \frac{\partial}{\partial y}\left(\frac{\partial c}{\partial y}\right)+\frac{\partial}{\partial z}\left(\mu_{v} \frac{\partial c}{\partial z}\right)\right)\right] d G= \\
=\iiint_{G}\left[\mu_{h} \frac{\partial}{\partial x}\left(c \frac{\partial c}{\partial x}\right)+\mu_{h} \frac{\partial}{\partial y}\left(c \frac{\partial c}{\partial y}\right)+\frac{\partial}{\partial z}\left(c \mu_{v} \frac{\partial c}{\partial z}\right)\right] d G-\iiint_{G}\left[\mu_{h}\left(\frac{\partial c}{\partial x}\right)^{2}+\mu_{h}\left(\frac{\partial c}{\partial y}\right)^{2}+\mu_{v}\left(\frac{\partial c}{\partial z}\right)^{2}\right] d G .
\end{gathered}
$$

Let $\vec{Q}=\left\{Q_{x}, Q_{y}, Q_{z}\right\}=\left\{\mu_{h} c \frac{\partial c}{\partial x}, \mu_{h} c \frac{\partial c}{\partial y}, c \mu_{v} \frac{\partial c}{\partial z}\right\}$. Then, by the Ostrogradsky-Gauss theorem, we have:

$$
\begin{gathered}
\iiint_{G}\left[\mu_{h} \frac{\partial}{\partial x}\left(c \frac{\partial c}{\partial x}\right)+\mu_{h} \frac{\partial}{\partial y}\left(c \frac{\partial c}{\partial y}\right)+\frac{\partial}{\partial z}\left(c \mu_{v} \frac{\partial c}{\partial z}\right)\right] d G=\iiint_{G} d i v \vec{Q} d G= \\
=\iint_{A A_{1} D_{1} D} Q_{y} d x d z+\iint_{D D_{1} C_{1} C} Q_{x} d y d z+\iint_{O B C C_{1}} Q_{y} d x d z+\iint_{A A_{1} O B} Q_{x} d y d z+\iint_{A B C D} Q_{z} d x d y+ \\
=\iint_{A_{1} O C_{1} D_{1}} Q_{z} d x d y=\iint_{A A_{1} D_{1} D} Q_{y} d x d z+\iint_{O B C C_{1}} Q_{y} d x d z+\iint_{A A_{1} O B} Q_{x} d y d z+\iint_{A B C D} Q_{z} d x d y .
\end{gathered}
$$

After transforming each term from the right-hand side of (14) taking into account the conditions on the boundary (3)-(5), we obtain

$$
\begin{gathered}
\iint_{A A_{1} D_{1} D} Q_{y} d x d z=\iint_{A A_{1} D_{1} D} c^{*} \mu_{h} \frac{\partial c^{*}}{\partial y} d x d z, \quad \iint_{D D_{1} C_{1} C} Q_{x} d y d z=\iint_{D D_{1} C_{1} C} c \mu_{h} \frac{\partial c}{\partial x} d y d z=0 \\
\iint_{O B C C_{1}} Q_{y} d x d z=\iint_{O B C C_{1}} c^{*} \mu_{h} \frac{\partial c^{*}}{\partial y} d x d z, \quad \iint_{A A_{1} O B} Q_{x} d y d z=\iint_{A A_{1} O B} c^{*} \mu_{h} \frac{\partial c^{*}}{\partial x} d y d z \\
\iint_{A B C D} Q_{z} d x d y=\iint_{A B C D} c \mu_{v} \frac{\partial c}{\partial z} d x d y=-\iint_{A B C D} c \mu_{v} \frac{w_{g}}{\mu_{v}} c d x d y=-\iint_{A B C D} c^{2} w_{g} d x d y=-\iint_{A B C D} w_{g} c^{2} d x d y \\
\iint_{A_{1} O C_{1} D_{1}} Q_{z} d x d y=\iint_{A_{1} O C_{1} D_{1}} c \mu_{v} \frac{\partial c}{\partial z} d x d y=0 .
\end{gathered}
$$

After substituting the obtained equalities in relation (14), we obtain

$$
\begin{gathered}
\iiint_{G}\left[\mu_{h} \frac{\partial}{\partial x}\left(c \frac{\partial c}{\partial x}\right)+\mu_{h} \frac{\partial}{\partial y}\left(c \frac{\partial c}{\partial y}\right)+\frac{\partial}{\partial z}\left(c \mu_{v} \frac{\partial c}{\partial z}\right)\right] d G= \\
=\iint_{A A_{1} D_{1} D} c^{*} \mu_{h} \frac{\partial c^{*}}{\partial y} d x d z+\iint_{O B C C_{1}} c^{*} \mu_{h} \frac{\partial c^{*}}{\partial y} d x d z+\iint_{A A_{1} O B} c^{*} \mu_{h} \frac{\partial c^{*}}{\partial x} d y d z-\iint_{A B C D} w_{g} c^{2} d x d y .
\end{gathered}
$$

Taking into account (11), (12), (13), and (15), the equality (10) takes the form 


$$
\begin{gathered}
\frac{1}{2} \iiint_{G} c^{2}(x, y, z, T) d G-\int_{0}^{T}\left(\int_{A A_{1} O B}\left(\frac{1}{2}\left(c^{*}\right)^{2} u+c^{*} \mu_{h} \frac{\partial c^{*}}{\partial x}\right) d y d z\right) d t- \\
-\int_{0}^{T}\left(\int_{B O C_{1} C}\left(\frac{1}{2}\left(c^{*}\right)^{2} v+c^{*} \mu_{h} \frac{\partial c^{*}}{\partial y}\right) d x d z\right) d t+\int_{0}^{T}\left(\iint_{A A_{1} D D_{1}}\left(\frac{1}{2}\left(c^{*}\right)^{2} v-c^{*} \mu_{h} \frac{\partial c^{*}}{\partial y}\right) d x d z\right) d t+\frac{3}{2} \int_{0}^{T}\left(\iint_{A B C D} w_{g} c^{2} d x d y\right) d t+(16) \\
+\int_{0}^{T}\left[\iint_{G} \int\left(\left(\frac{\partial c}{\partial x}\right)^{2}+\mu_{h}\left(\frac{\partial c}{\partial y}\right)^{2}+\mu_{v}\left(\frac{\partial c}{\partial z}\right)^{2}\right) d G\right] d t=\frac{1}{2} \iiint_{G} c^{2}(x, y, z, 0) d G+\iint_{0}^{T}\left(\iiint_{G} c F d G\right) d t .
\end{gathered}
$$

The identity (16) will be the main one in investigating the uniqueness and obtaining an a priori estimate of the norm of the solution of the initial-boundary value problem (1)-(5). If the boundary condition (5) is replaced by the boundary condition (6), the quadratic functional (17) changes as follows:

$$
\begin{gathered}
\frac{1}{2} \iiint_{G} c^{2}(x, y, z, T) d G-\iint_{0}^{T}\left(\int_{A A_{1} O B}\left(\frac{1}{2}\left(c^{*}\right)^{2} u+c^{*} \mu_{h} \frac{\partial c^{*}}{\partial x}\right) d y d z\right) d t- \\
-\int_{0}^{T}\left(\int_{B O C_{1} C}\left(\frac{1}{2}\left(c^{*}\right)^{2} v+c^{*} \mu_{h} \frac{\partial c^{*}}{\partial y}\right) d x d z\right) d t+\int_{0}^{T}\left(\iint_{A A_{1} D D_{1}}\left(\frac{1}{2}\left(c^{*}\right)^{2} v-c^{*} \mu_{h} \frac{\partial c^{*}}{\partial y}\right) d x d z\right) d t+ \\
+\int_{0}^{T}\left(\iint_{A B C D}\left(\frac{1}{2} w_{g}-\alpha \mu_{v}\right) c^{2} d x d y\right) d t+\int_{0}^{T}\left[\iiint_{G}\left(\left(\frac{\partial c}{\partial x}\right)^{2}+\mu_{h}\left(\frac{\partial c}{\partial y}\right)^{2}+\mu_{v}\left(\frac{\partial c}{\partial z}\right)^{2}\right) d G\right] d t= \\
=\frac{1}{2} \iiint_{G} c^{2}(x, y, z, 0) d G+\iint_{0}^{T}\left(\iiint_{G} c F d G\right) d t .
\end{gathered}
$$

Let suppose that equation (1) with the same conditions (2)-(5) is satisfied by two different solutions of problem $c_{1}=c_{1}(x, y, z, t), c_{2}=c_{2}(x, y, z, t)$. For their difference, the following initial-boundary value problem may be formulated:

$$
\begin{gathered}
\frac{\partial \tilde{c}}{\partial t}+\frac{\partial(u \tilde{c})}{\partial x}+\frac{\partial(v \tilde{c})}{\partial y}+\frac{\partial\left(\left(w+w_{g}\right) \tilde{c}\right)}{\partial z}=\mu_{h}\left(\frac{\partial^{2} \tilde{c}}{\partial x^{2}}+\frac{\partial^{2} \tilde{c}}{\partial y^{2}}\right)+\frac{\partial}{\partial z}\left(\mu_{v} \frac{\partial \tilde{c}}{\partial z}\right), \\
\tilde{c}(x, y, z, 0)=0,(x, y, z) \in \bar{G},
\end{gathered}
$$

- on the faces $A A_{1} O B, A A_{1} D_{1} D, B O C_{1} C, D D_{1} C_{1} C, A_{1} O C_{1} D_{1}$

$$
\tilde{c}=c^{*}-c^{*}=0 ;
$$

- on the bottom surface $A B C D$

$$
\frac{\partial \tilde{c}}{\partial z}=-\frac{w_{g}}{\mu_{v}}\left(c_{1}-c_{2}\right)=-\frac{w_{g}}{\mu_{v}} \tilde{c}
$$

For a function $\tilde{c}$ (17) takes the form taking into account equalities (18)-(21)

$$
\frac{1}{2} \iiint_{G} \tilde{c}^{2}(x, y, z, T) d G+\frac{3}{2} \int_{0}^{T}\left(\iint_{A B C D} w_{g} \tilde{c}^{2} d x d y\right) d t+\int_{0}^{T}\left[\iiint_{G}\left[\mu_{h}\left(\frac{\partial \tilde{c}}{\partial x}\right)^{2}+\mu_{h}\left(\frac{\partial \tilde{c}}{\partial y}\right)^{2}+\mu_{v}\left(\frac{\partial \tilde{c}}{\partial z}\right)^{2}\right] d G\right] d t=0
$$


Because the $w_{g}>0$ and other known terms under the integral sign are positive $\mu_{h}>0, \mu_{v}>0$, then (22) is satisfied under condition

$$
\tilde{c}(x, y, z, t) \equiv 0,(x, y, z) \in G, 0<t \leq T,
$$

which completes the proof of the uniqueness of the solution of the initial boundary value problem (1)-(5).

In the case of changing (5) to the boundary condition (6), instead of relation (22), we obtain appropriate equality in the form

$$
\frac{1}{2} \iint_{G} \tilde{c}^{2}(x, y, z, T) d G+\int_{0}^{T}\left(\iint_{A B C D}\left(\frac{1}{2} w_{g}-\alpha \mu_{v}\right) \tilde{c}^{2} d x d y\right) d t+\int_{0}^{T}\left[\iiint_{G}\left[\mu_{h}\left(\frac{\partial \tilde{c}}{\partial x}\right)^{2}+\mu_{h}\left(\frac{\partial \tilde{c}}{\partial y}\right)^{2}+\mu_{v}\left(\frac{\partial \tilde{c}}{\partial z}\right)^{2}\right] d G\right] d t=0 .
$$

If we require the fulfillment of inequality

$$
\frac{1}{2} w_{g}-\alpha \mu_{v} \geq 0,(x, y, z) \in A B C D, 0<t \leq T \text { or } \alpha \leq \frac{w_{g}}{2 \mu_{v}},(x, y, z) \in A B C D, 0<t \leq T,
$$

then all the terms in (24) are non-negative and the equality to zero is possible if and the only if $\tilde{c}(x, y, z, t) \equiv 0,(x, y, z) \in G, 0<t \leq T$, which means that the solution is unique in this case.

Theorem. Suppose that equation (1)

$$
\frac{\partial c}{\partial t}+\frac{\partial(u c)}{\partial x}+\frac{\partial(v c)}{\partial y}+\frac{\partial\left(\left(w+w_{g}\right) c\right)}{\partial z}=\mu_{h}\left(\frac{\partial^{2} c}{\partial x^{2}}+\frac{\partial^{2} c}{\partial y^{2}}\right)+\frac{\partial}{\partial z}\left(\mu_{v} \frac{\partial c}{\partial z}\right)+F
$$

in a simply connected domain $\Omega_{T}=G \times(0<t<T), G=\left(0<x<L_{x}, 0<y<L_{y}, 0<z<H(x, y)\right)$, with a sufficiently smooth boundary defined by the smoothness of the function $z=H(x, y)$, $0 \leq x \leq L_{x}, 0 \leq y \leq L_{y}$ with the initial and boundary conditions (2)-(5). Let the solution functions $c(x, y, z, t)$ velocity vector of the water environment $\left\|u, v, w+w_{g}\right\|^{T}$, initial condition $c_{0}(x, y, z)$, right-hand side $F(x, y, z, t)$, boundary condition $c^{*}(x, y, z, t)$, the coefficient of vertical turbulent exchange $\mu_{v}=\mu_{v}(z),(x, y, z) \in G$ satisfy the following smoothness conditions: $c(x, y, z, t) \in C^{2}\left(\Omega_{T}\right) \cap C\left(\bar{\Omega}_{T}\right), \quad \operatorname{grad} c \in C\left(\bar{\Omega}_{T}\right), \quad\left\|u, v, w+w_{g}\right\|^{T} \in C^{(1)}\left(\Omega_{T}\right) \cap C\left(\bar{\Omega}_{T}\right)$, $c_{0}(x, y, z) \in C(\bar{G}), \quad F(x, y, z, t) \in C\left(\Omega_{T}\right), \quad \mu_{v}(x, y, z) \in C^{(1)}(G) \cap C(\bar{G}), \quad c^{*}(x, y, z, t) \in C(S) \times[0 \leq t \leq T]$, $c^{*}(x, y, z, t) \in C(S) \times[0 \leq t \leq T], S=\bar{G} \backslash G, \quad \frac{\partial c^{*}}{\partial n} \in C\left(\left(0 \leq x \leq L_{x}, 0 \leq y \leq L_{y}, z=H(x, y)\right) \times[0 \leq t \leq T]\right), \quad$ and also the conditions for the consistency of the boundary and initial conditions, $c^{*}(x, y, z, 0)=c_{0}(x, y, z), \quad(x, y, z) \in S \backslash\left(0<x<L_{x}, 0<y<L_{y}, z=H(x, y)\right), \quad \frac{\partial c_{0}}{\partial z}=-\frac{\mu_{v}}{w_{g}} c^{*}$, $\left(0<x<L_{x}, 0<y<L_{y}, z=H(x, y)\right)$, then the solution of this problem exists and is unique.

Comment. If the boundary condition (5) is replaced by the boundary condition (6), inequality (25) should be added as a sufficient condition for the fulfillment of the preceding theorem. 


\section{Investigation of the continuous dependence of the solutions of the initial-boundary value problem of slurry transport from boundary, initial conditions and the function of the right-hand side}

The next stage is connected with the study of the continuous dependence of the solution on the functions: the right-hand side, the boundary and initial conditions for equation (1).

We assume that

$$
c^{*} \geq c_{0}^{*} \equiv \text { const }>0,0 \leq x \leq L_{x}, 0 \leq y \leq L_{y}, 0 \leq z \leq L_{z}, 0 \leq t \leq T .
$$

We introduce for convenience the notation: the union of all parts of a lateral cylindrical surface - the boundary of the domain $G$ denoted by $S_{c}$, and the lower base of the region $G-S_{b}$. By virtue of the smoothness conditions listed under the conditions of the theorem, extrema of functions on bounded closed sets are attained:

$$
\begin{gathered}
M_{1} \equiv \max _{\Omega_{T}}\{|c|\}, \quad M_{2} \equiv \max _{S}\left\{\left|\frac{\partial c}{\partial x}\right|,\left|\frac{\partial c}{\partial y}\right|\right\}, \\
M_{3} \equiv \max _{S_{c}}\left\{\mu_{h}\right\}, \quad M_{4} \equiv \max _{S_{c} \times[0 \leq t \leq T]}\{|u|,|v|\}, \quad M_{5} \equiv \min _{G}\left\{\mu_{h}, \mu_{v}\right\} .
\end{gathered}
$$

We will orient to equality (16) if the boundary condition (5) is used, and equality (17)in case of boundary condition (6). Using the Friedrichs inequality, we have a chain of inequalities:

$$
\begin{aligned}
\iiint_{G}\left(\mu_{h}\left(\frac{\partial c}{\partial x}\right)^{2}+\mu_{h}\left(\frac{\partial c}{\partial y}\right)^{2}\right. & \left.\left.+\mu_{v}\left(\frac{\partial c}{\partial z}\right)^{2}\right) d G \geq \min _{G}\left\{\mu_{h}, \mu_{v}\right\} \iiint_{G} \int\left(\frac{\partial c}{\partial x}\right)^{2}+\left(\frac{\partial c}{\partial y}\right)^{2}+\left(\frac{\partial c}{\partial z}\right)^{2}\right) d G \geq \\
& \geq M_{5}\left(\pi^{2}\left(\frac{1}{L_{x}^{2}}+\frac{1}{L_{y}^{2}}+\frac{1}{L_{z}^{2}}\right)\right) \iiint_{G} c^{2} d G
\end{aligned}
$$

Let us consider equation (16), from which, using (27) and (28) formulas, we obtain the inequality:

$$
\begin{gathered}
\iiint_{G}\left(c^{2}(x, y, z, T)\right) d G+2 M_{5}\left(\pi^{2}\left(\frac{1}{L_{x}^{2}}+\frac{1}{L_{y}^{2}}+\frac{1}{L_{z}^{2}}\right)\right) \int_{0}^{T} \int\left(\iint_{G}\left(c^{2}(x, y, z, \mathrm{t})\right) d G\right) d t+ \\
+3 \iint_{0}^{T}\left(\iint_{S_{b}} w_{g} c^{2} d x d y\right) d t \leq \iiint_{G}\left(c_{0}^{2}(x, y, z)\right) d G+M_{4} \iint_{0}^{T}\left(\iint_{S_{c}}\left(c^{*}\right)^{2} d S\right) d t+ \\
+2 M_{2} M_{3} \int\left(\iint_{0}^{T}\left|c_{S_{c}}^{*}\right| d S\right) d t+2 M_{1} \int\left(\iiint_{0}^{T}|F| d G\right) d t .
\end{gathered}
$$

The inequality (29) implies two inequalities

$$
\begin{gathered}
\iiint_{G}\left(c^{2}(x, y, z, T)\right) d G \leq \\
\leq \iiint_{G} c_{0}^{2} d G+M_{4} \int_{0}^{T}\left(\iint_{S_{c}}\left(c^{*}\right)^{2} d S\right) d t+2 M_{2} M_{3} \int_{0}^{T}\left(\iiint_{S_{c}}\left|c^{*}\right| d S\right) d t+2 M_{1} \int_{0}^{T}\left(\iiint_{G}|F| d G\right) d t .
\end{gathered}
$$


and

$$
\begin{gathered}
\int_{0}^{T}\left(\iiint_{G}\left(c^{2}(x, y, z, T)\right) d G\right) d t \leq \\
\leq M_{6}\left(\iiint_{G} c_{0}^{2} d G+M_{4} \int_{0}^{T}\left(\iint_{S_{c}}\left(c^{*}\right)^{2} d S\right) d t+2 M_{2} M_{3} \int_{0}^{T}\left(\iint_{S_{c}}\left|c^{*}\right| d S\right) d t+2 M_{1} \int_{0}^{T}\left(\iiint_{G}|F| d G\right) d t\right) .
\end{gathered}
$$

where $M_{6}=\frac{1}{2 M_{5}}\left(\pi^{2}\left(\frac{1}{L_{x}^{2}}+\frac{1}{L_{y}^{2}}+\frac{1}{L_{z}^{2}}\right)\right)^{-1}$.

From these inequalities follows the continuous dependence (stability) of the solution of problem (1)-(5) on functions: the initial condition, the boundary conditions and the righthand side, in the norm $L_{2}$ for any moment of time $0<T<+\infty$, and also in the time-integral norm $L_{2}$.

Obviously, if the inequality (27) is satisfied, the initial boundary value problem (1)-(4), (6), when the conditions of the theorem in p.2 is satisfied, also have a solution depending continuously on the initial condition, boundary conditions, and right conditions parts in appropriate norms.

\section{Conclusion}

Three-dimensional mathematical model of transport of bottom material in coastal systems which satisfies the basic conservation laws has been studied. The novelty of the results is that for the proposed mathematical model which describes the motion of suspended particles in three directions, taking into account the following physical processes and parameters: diffusion-convection, complicated bottom and shoreline geometry, water currents, lifting and sedimentation sufficient uniqueness and stability conditions for two typical bottom boundary conditions have been obtained. Conditions for the uniqueness of the solution of the initial-boundary value problem are formulated in the form of corresponding theorem as well as appropriate inequalities have been obtained, which guarantee the stability of the solution of initial-boundary value problem on functions: the initial condition, the boundary conditions and the right-hand side, in the norm $L_{2}$ for any moment of time $0<T<+\infty$, and also in the time-integral norm $L_{2}$.

This paper was partially supported by the grant No.17-11-01286 of the Russian Science Foundation.

\section{References}

1. A.A. Sukhinov, A.I. Sukhinov, Parallel Computational Fluid Dynamics, Mutidisciplinary Applications, Proceedings of Parallel CFD 2004 Conference, 223 (2005) DOI: 10.1016/B978-044452024-1/50029-4.

2. A.I. Sukhinov, A.A. Sukhinov, Parallel Computational Fluid Dynamics, Multidisciplinary Applications, Proceedings of Parallel CFD 2004 Conference, 231 (2005) DOI: 10.1016/B978-044452024-1/50030-0.

3. P.L. Barnard, B.E. Jaffe, and D.H. Schoellhamer, Preface for special issue of Marine Geology, in Barnard, P.L., Jaffe, B.E., and Schoellhamer, D.H., eds., A multidiscipline approach for understanding sediment transport and geomorphic evolution in 
an estuarine-coastal system-San Francisco Bay: Marine Geology, 345, 1, (2013) doi:10.1016/j.margeo.2013.09.010.

4. A.I. Sukhinov, A.E. Chistyakov, E.A. Protsenko, Mathematical Models and Computer Simulations., 6, 4 , 351 (2014) DOI: https://doi.org/10.1134/S2070048214040097

5. A.I. Sukhinov, A.E. Chistyakov, A.V. Shishenya, E.F. Timofeeva, Mathematical Models and Computer Simulations, 5, 2, 122 (2013) DOI: https://doi.org/10.1134/S2070048213020087

6. V.V. Sidoryakina, A.I. Sukhinov, Computational Mathematics and Mathematical Physics, 57:6, 978 (2017) DOI: https://doi.org/10.7868/S0044466917060138.

7. A.I. Sukhinov, V.V. Sidoryakina, A.A.Sukhinov, Vestnik of Don State Technical University, 1 (88), 5 (2017)

8. A. Sukhinov, V. Chistyakov, V. Sidoryakina, MATEC Web Conf, 132, 04003 (2017)

9. A.I. Sukhinov, V.V. Sidoryakina, Andrey A. Sukhinov, Computational Mathematics and Information Technologies, 1, 1, 21 (2017)

10. A.I. Sukhinov, V.V. Sidoryakina, Math. Modelling., 29, 11, 19 (2017)

11. V.A. Ladyzhenskaya, N.N. Solonnikova, Ural'tsova Linear and quasilinear equations of parabolic type (Nauka, Moscow, 1967)

12. M.H. Protter, H.F. Weinberger, Maximum Principles in Differential Equation. (Springer-Verlag, New York, 1984) 